\title{
Conceptualizing the evolution of Tmor-Da
}

\section{SIGRADI2018 TECHNOPOLITICAS \\ xxii congresso da sociedade iberoamericana de gráfica digital 22th conference of the iberoamerican society of digital graphics 07|08|09|novembro|2018 iau usp | são carlos $\mid \mathrm{sp}$ br}

\author{
Yee Kee Ku \\ The University of Melbourne | Australia | yku@student.unimelb.edu.au \\ Michael Kirley \\ The University of Melbourne | Australia | mkirley@unimelb.edu.au \\ Justyna Karakiewicz \\ The University of Melbourne | Australia | justynak@unimelb.edu.au
}

Yi Mo Jiang

The University of Melbourne | Australia | yimo.jiang@alumni.unimelb.edu.au

\begin{abstract}
The urban dynamics observable in an informal settlement are akin to the characteristics of a complex adaptive system. In this paper, we describe an Agent-Based Model designed to simulate the urban dynamics of an informal settlement, Tmor-Da, in Phnom Penh, Cambodia. The overarching goal was to understand the possible rules that guided the development of built forms and the use of residue spaces in Tmor-Da. A series of simulation experiments were used to examine alternative hypotheses derived from field work and desktop analysis related to morphological changes associated with spatial units, including homes, shops, the orphanage, church and temple. The results suggest that the complex, emergent patterns encapsulated within the informal settlement could be reproduced in a simulation model. We conclude that our model can be used as an investigative tool to explore the most plausible factors contributing to the evolutionary trajectory of an informal settlement.
\end{abstract}

Keywords: Informal settlement; Urban evolution; Urban morphology; Agent-based model; Complex adaptive system.

\subsection{INTRODUCTION}

Informal settlements are often characterised by their ability to adapt to continually changing conditions, as such that they are a quintessential example of a complex adaptive system [CAS] (Batty, 2013, Holland, 2012). Urban morphology techniques (Marcos, 2012; Oliveira, 2016) provide a systematic approach to further our understanding of the rules guiding the development of built forms and the use of remaining spaces in such communities. However, informal settlements are often undocumented or excluded from official maps (Patel \& Baptist, 2012). Consequently, building robust simulation models of the urban morphology of informal settlements is challenging.

In this paper, we examine the morphological changes of Tmor-Da, an inner city informal settlement in Phnom Penh, Cambodia. Tmor-Da was chosen as a case study because: (1) the inhabitants predominantly occupy abandoned structures and destroyed buildings before expanding into the entire area; (2) inhabitants are continually adapting and readapting the existing spaces for various activities. Such behaviours have impacted on the observed variation in private housing, shop-houses and shared public spaces alike to laneways and squares.
We describe the design and implementation of an AgentBased Model (ABM) of the evolution of Tmor-Da. The general modelling approach was similar to the framework used in related studies (Patel, Crooks, \& Koizumi, 2018) (Fisher-Gewirtzman \& Blumenfeld-Liberthal, 2012), and is consistent with Holland's approach for modelling complex adaptive systems (Holland, 2010). We combined GIS theme layers and individual-based modelling techniques to create an artificial, spatial-heterogeneous world with multiscale interactions and non-linear feedbacks loops. Rules governing the structure of built form and the use of space were generated from field studies including observations, on-site measurements, interpolated population data, old cartographic maps and current aerial photographs.

Simulation experiments were used to test hypotheses designed to explore the rule-based scenarios guiding morphological changes over the period 1979-2016. The results show that the simulation outcomes are consistent with fieldwork observations, suggesting that our $A B M$ is a robust tool that can be used to explore the most plausible factors contributing to the evolutionary trajectory in TmorDa.

\subsection{BACKGROUND \\ 2.1 INFORMAL SETTLEMENT}

The motivation behind studying the urban dynamics of informal settlement is to better understand how the 
'system' as a whole adapts to unforeseen changes over time. These changes may be social, economic, physical developments or triggered by natural disasters (Sennett, 1992). Thus, the goal is to understand the resilient qualities of the system.

Inherently, informal settlements are natural human habitats built by a group of dwellers for themselves. First, they selfbuild shelters with/ between each other and live as neighbours. Over time, as they 'earn and learn', the dwellers improve their shelters into more permanent dwellings. Some expand the area of their dwellings or land, negotiating their area between their immediate neighbours (Habraken, 1998). This action is continuous in a series of cycles, and the reconfiguration process for the dwellings influence the evolution of the informal settlement's built forms. As the neighbourhood grows without any authoritative control over this process, the interactions in a neighbourhood become more complicated due to collective behaviours. Nonetheless, the simple logic of the interactions remains between immediate neighbours. Consequently, the entire informal settlement evolves in a self-organised manner and under some circumstance may generate an unpredictable, irregular and possibly chaotic urban structure (Barros, 2008).

\subsection{COMPLEX ADAPTIVE SYSTEM}

The study of complex adaptive systems stems from complexity theory, which describes the intricate relations between a system and its environment, and the elements within a system (Gell-Mann, 1994). The characteristics of complex adaptive systems are typically listed as; adaptation (Holland, 1975), emergence and selforganisation (Allen, 1997), complex collective behaviour based on simple rules, unpredictability (Allen \& Strathern, 2005), novelty and robustness (Allen, 2014), connectivity through signalling and information processing (Bettencourt \& West, 2010), and history (Karakiewicz, 2016). In the urban environment, complex adaptive systems consist of large systems of interacting components that through nonlinearity, may produce emergent phenomenon and selforganised structure (Batty, 2005).

Informal settlements are a natural human ecosystem that are apart of a city and eventually the broader ecology. The evolution of an informal settlement can be studied as a small set of critical processes that create and maintain selforganisation (Gunderson \& Holling, 2002). If we examine the dynamics of informal settlements through the lens of the complex adaptive system, we can explore how the settlements maintain their structural coherence via adaptation (Holland, 2010). The importance of understanding these critical processes is to learn about the adaptive behaviour that supports the resilience of the system. Here, adaptation is observed as the flexibility of the dwellers manifested and manifesting in the built forms of the informal settlement.

\section{3}

\section{RELATED WORK}

A number of models that combine GIS theme layers and individual-based modelling techniques to create an artificial, spatial-heterogeneous world with multi-scale interactions and non-linear feedbacks loops have been reported in the literature. Two particular models are relevant to this study:

The first model was a Spatial Agent-based Modelling [SAM]; a multi-scalar framework benefitted from the integration of Geographic Information System [GIS] with ABM. Taking a slum area at Ahmedabad, India as a case study, the emergent structure of the slum was modelled. This dynamic model integrated the GIS as the spatial environment in the city for housing market at the macro level, and merged with an ABM simulation of interactions between agents at the micro level. Agents were modelled as two main types: slum inhabitants, and local government. The generated outputs were evaluated using spatial analyses techniques provided through GIS's analysis of the empirical data (Patel, Crooks, \& Koizumi, 2018).

The second relevant model was an Agent-based Cellular Automata [ACA] built to study an ancient Arab settlement in Sachin, Galilee in Northern Israel. The urban morphology of the settlement has no apparent continuity, and the alignment of the settlement did not conform to any strict geometrical patterns or streets. The ACA modelled the formation process and discovered that the bottom-up driving force was by individual agents that did not obey topdown planning rules. Those findings verified that the settlement emerged from cultural and social behaviours (Fisher-Gewirtzman \& Blumenfeld-Liberthal, 2012).

\subsection{THE MODEL}

\subsection{HIGH-LEVEL DESCRIPTIONS}

Our ABM was designed to simulate the urban morphology of Tmor-Da is an individual-based model, where we combine cellular automata, GIS theme layers and agent concepts. The GIS layers provide the structure for the urban environment, which are mapped to the cells of a regular lattice of a $2 \mathrm{D}$ cellular automata. We extend this framework to model spatial units - built forms and voids represented as interacting agents. Iterations of the transition rules provide the scaffolding for spatial units to diffuse across the lattice and for 3D structures (represented as multiple cubes) to emerge. We implement the model using GAMA (version 1.7), a general ABM simulation platform (http://gama-platform.org).

\subsection{CASE STUDY: TMOR-DA}

Preliminary fieldwork was carried out in the year 2016. Data collected relevant to Tmor-Da spanned from the years 1889 until 2016. The data collated included: photographs of the site; measurements of existing building dimensions; old cartographic maps; limited satellite images; interpolated population data; and observations of urban dynamics of the dwellers. 
Tmor-Da's site boundary during the French Protectorate era was demarcated by the surrounding roads (based on a 1950s-map obtained from National Archives of Cambodia). At this time, the Sister of Providences Institute [SPI] (a Catholic denomination) occupied the site. A map from the 1920s shows the existence of Cambodian wat and Chinese Cantonese temple at the site. The Cambodian wat is still intact and functioning. However, the temple and SPI were destroyed by the Khmer Rouge between 1974 to 1979. The orphanage and church were left intact and used as living quarter and head office.

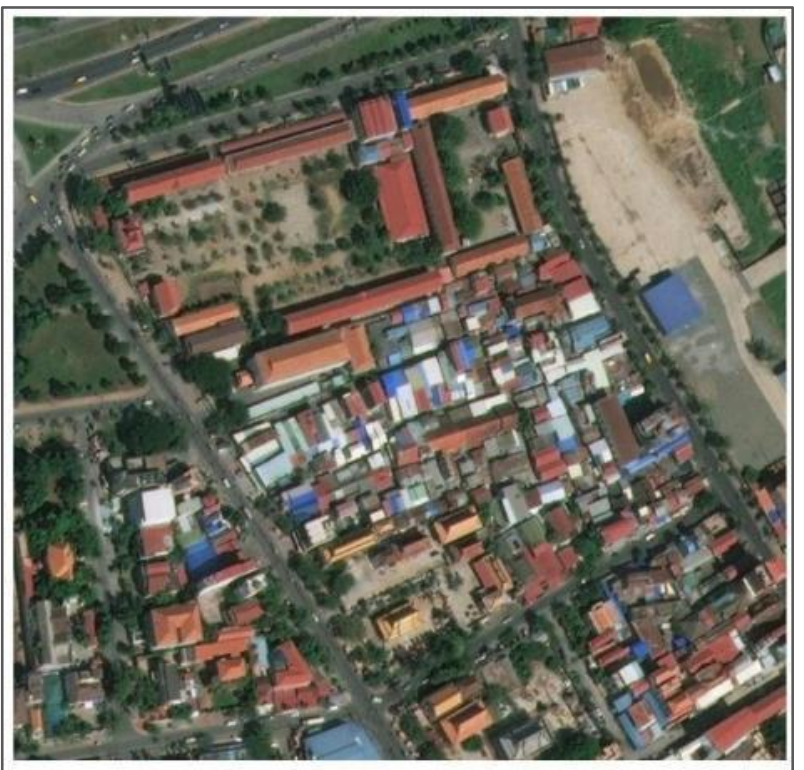

Figure 1: A satellite aerial image of Tmor-Da from 2016. Source: Authors.

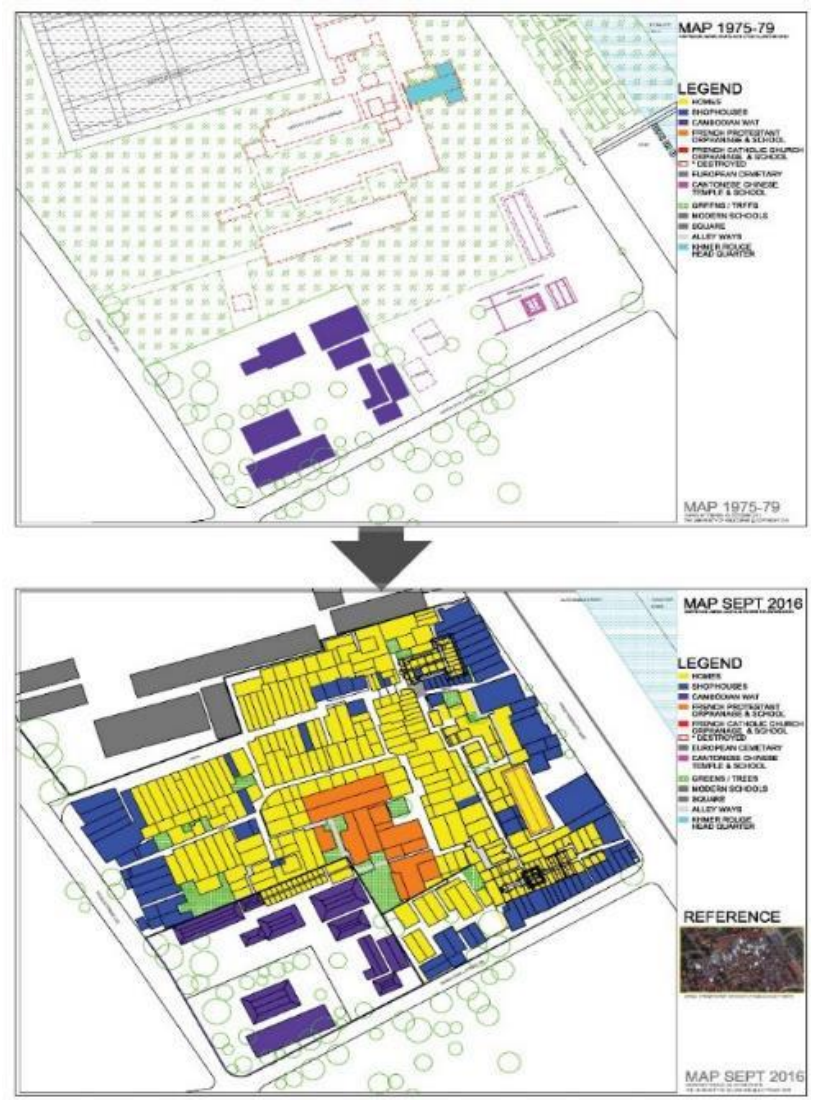

Figure 2: Two maps identified as the beginning of the informal settlement in year 1979 (top) and year 2016 (bottom). Source: Authors.
The informal settlement of Tmor-Da first emerged after the Khmer Rouge left Phnom Penn in 1979. The current dwellings were built into the remaining walls and roofs of the destroyed structures. Interestingly, similar informal structures can be observed in the church. Dwellings were also built over the orphanage's existing building, while the orphanage extended further into the central site area closer to the Cambodian Wat.

We observed that the informal settlement has expanded within the site boundary, and the built form along the site perimeter have evolved to become shop-houses. Over the years, these dwellings (approximately three meters by four meters in size) have gentrified from shacks into permanent homes, with the residents attaining legal ownership of the properties in the 1980s. Today, most of the ground area of the built form on the site is occupied.

Field observations suggest that the settlement is constantly evolving. For example, some homes have expanded vertically into two and three floors above. However, the formation process and empirical data related to population growth and the types of business have not been documented. Unfortunately, many existing documents related to Tmor-Da were destroyed.

\subsection{MODEL DETAILS \\ 3.3.1 SPATIAL UNITS}

An essential first step in designing the ABM was to identify key spatial units, which can be classified as types of built form and residue spaces. Figure 3 lists the individual spatial units, represented by specific colours (attributes). These spatial units were mapped to cells of a cellular automaton's regular lattice. 2D cells refer to the footprint of previous buildings: SIP (red), church (maroon), temple (magenta) and orphanage (orange). The other solid spatial units were homes (yellow), shop-houses (blue), wat (purple) and schools (brown). The void categorised the residual spaces represented by $2 \mathrm{D}$ cells for square (beige), paths (or laneways in greys), main roads (black line), cemetery (dark grey) and greens (green). Green areas were the bushes, grass and wild plants. It should be noted that 3D structures (represented as multiple cubes) can also be mapped to the cell positions on the cellular automaton's lattice.

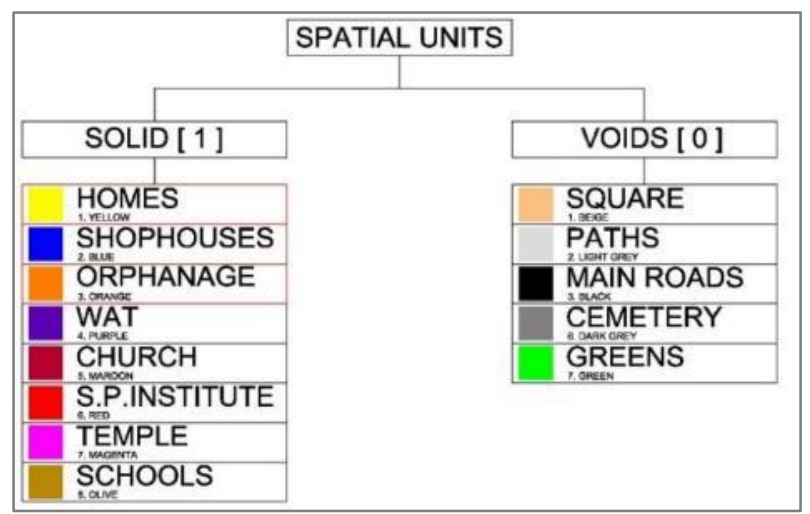

Figure 3: Spatial units. Source: Authors. 


\subsubsection{MODEL PARAMETERS}

A range of parameters are used to control model dynamics, these include: local neighbourhood size and topology; interaction probability values between specific spatial units (cells and cubes); and probability values associated with the transition rules (i.e. rules guiding the morphology of the settlement); and the time steps used in the simulation. Figure $4 \mathrm{a}$ lists the parameter values from the GAMA platform window. The user can set the parameter values via sliders/toggle bars. It should be noted, that minor differences in the magnitude of specific input parameters may generate very different evolutionary trajectories of the urban morphology.

\subsubsection{TRANSITION RULES}

The transitional rules encoded into the model encapsulate two significant forces in the urban environment: top-down enforced actions (e.g., urban regulations, policies or buildings by authorities), and bottom-up interactions generated by dwellers. Figure 6 provides examples of these rules.

One example of a top-down rule is illustrated in GIS theme layers shown in Figure 5. For example, at a time step corresponding to the year 1989, we initialised a layer of brown cells and cubes, which represents the government school at the north end of the site. The school was built on the site of the grey cells (European cemetery), red (SPI footprint) and green cells read-in the model from previous time steps. Another top-down rule implements the minor changes to the main road configuration, which enlarged the north-east's site boundary.

One example of a bottom-up rule is related to the paths or laneways. Given the lack of historical data describing the emergent network of pathways, we developed a fractal model to grow the network generated by attracting major to minor pedestrian links (Batty, 2013; Batty \& Longley, 1994). Here, the laneways were classified based on the major pedestrian link as the main laneways, followed by two other sub-links and the minor ones are the cul-de-sacs. Each type of laneway was referenced as a static layer at an interval of 10 years' time-steps, starting from the main laneways.

An essential bottom-up rule associated with the morphogenesis of the built forms (see Figure 6) guides the transitional relationship between homes (yellow), shops (blue) orphanage(orange).

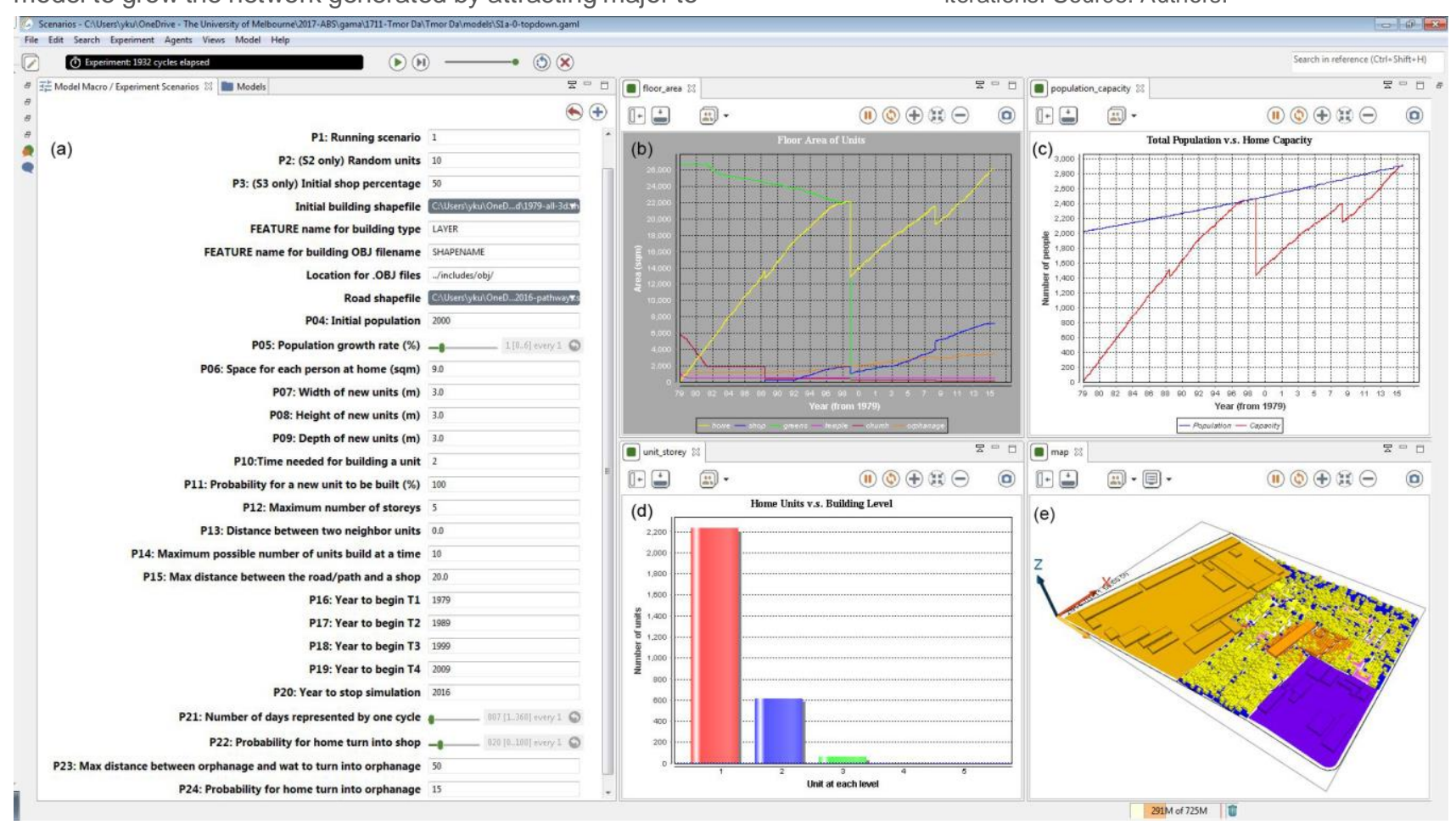

Figure 4: User interface screen on GAMA (V1.7) platform:

(b) Time-series plot of spatial units

(a) List of model parameters (P1 - P24) with adjustable magnitudes.

(d) graph of home units vs building level.

(c) Time-series plot of total population vs. home capacity

(e) Simulated urban pattern for scenario 1. Source: Authors. 


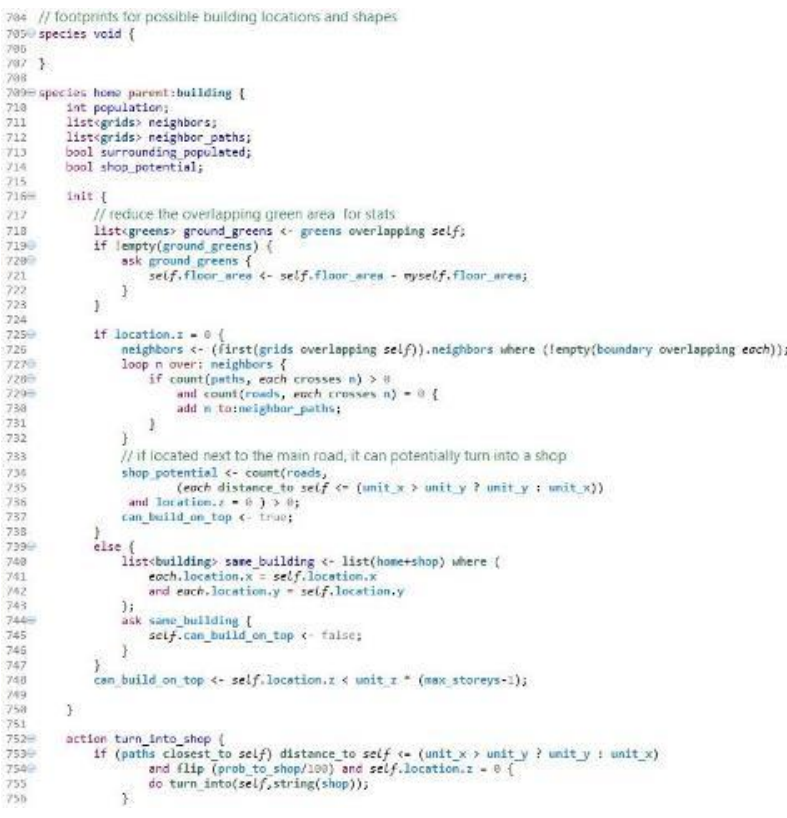

(a) Transitional rules encoded for scenario 1

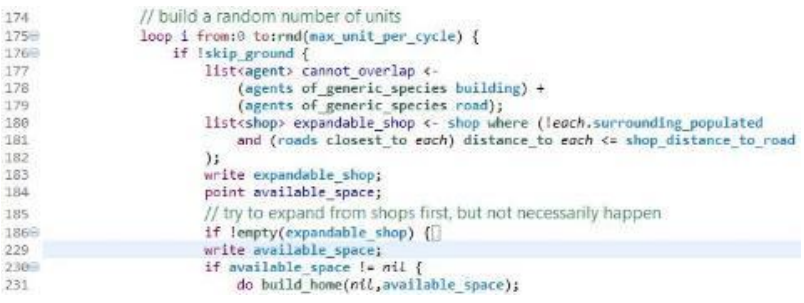

(b) Transitional rules encoded for scenario 2

Figure 6: Transitional rules and model parameters input for scenario 1 and 2. Source: Authors.

\subsection{SIMULATIONS AND RESULT}

To evaluate the efficacy of our urban evolutionary model, we propose two different scenarios describing the transition of built forms. The model parameters and transition rules corresponding to each of the scenarios are listed in Figure 6 . Each simulation trial was run for a fixed number of time steps (representing 1979-2016) from the same initial conditions. We describe the scenarios and present qualitative results before presenting statistical comparisons between the two scenarios.

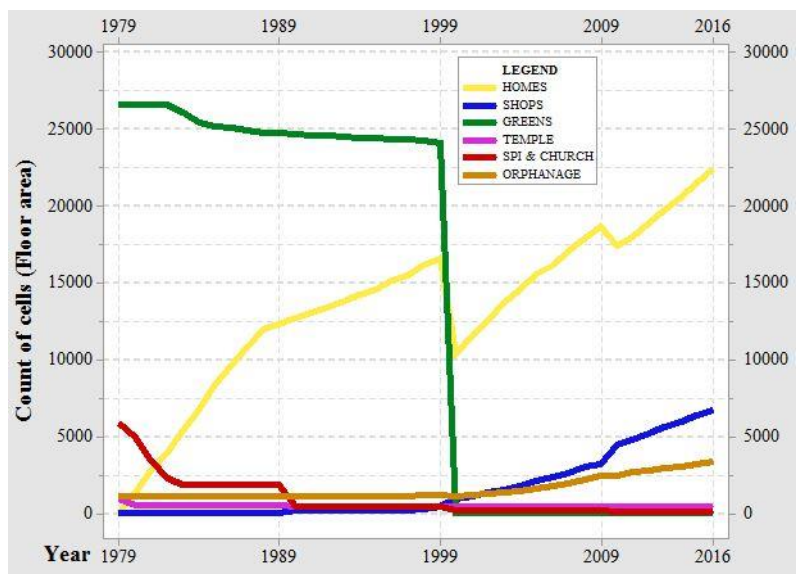

Figure 7: Graph of scenario 1's time-series plot for each spatial unit's floor areas (Year 1979-2016). Source: Authors.

\subsubsection{SCENARIO 1}

In the first scenario, our hypothesis assumes that homes occupy the existing abandoned buildings and destroyed structures first, before expanding to the nearby land. The footprint of the magenta (Chinese temple) and red (SPI) cells embedded in the cellular automata lattice were initialised with yellow cubes (houses). The local von Neumann neighbourhood size was set to 1 unit. The probability of the yellow cubes expanding to occupy the magenta cells and red cubes and before occupying their neighbourhood green cells was set to $30 \%$. After a number of time steps, the yellow (homes) will change to blue (shops), simulating the evolutionary dynamics (Figure 6a).

The simulation output is shown in Figure 9 (left). An inspection of the figure reveals a very similar pattern to the actual patterns observed on site in the year 2016 (Figure 2bottom). At the end of the simulation, the number of yellow (home), blue (shop) and orange (orphanage) have increased (Figure 7). Based on this qualitative observation and quantitative analysis, we can suggest that this hypothesis may be the main driving force responsible for the expansion of homes across the informal settlement.

\subsubsection{SCENARIO 2}

In the second scenario, our hypothesis assumes that the shop-houses at the site boundary provide the main driving force that impacts on the development of the entire informal settlement. The shops (blue) along the main road were assigned as 'attractors,' which encouraged the building of homes (yellow) towards the centre of the site. The local Von Neumann neighbourhood size was set to 1 unit. The probability of the yellow cubes expanding to occupy the magenta cells and red cubes, and before occupying their neighbourhood green cells was set to $30 \%$. Based on a probability value of $50 \%$, shops can 'expand' and seed the building of new homes within neighbourhood up to 10 cells from the site boundary's grid. Subsequent expansion of the yellow cubes following the transition rules described earlier (see Figure 6b).

The simulation output is shown in Figure 9(right). It should be noted that expansion of the houses stops after 2006 as the conditions do not allow for any new dwellings to occupy vacant space. At the end of the simulation, the number of yellow (home) and blue (shop) have increased at almost a

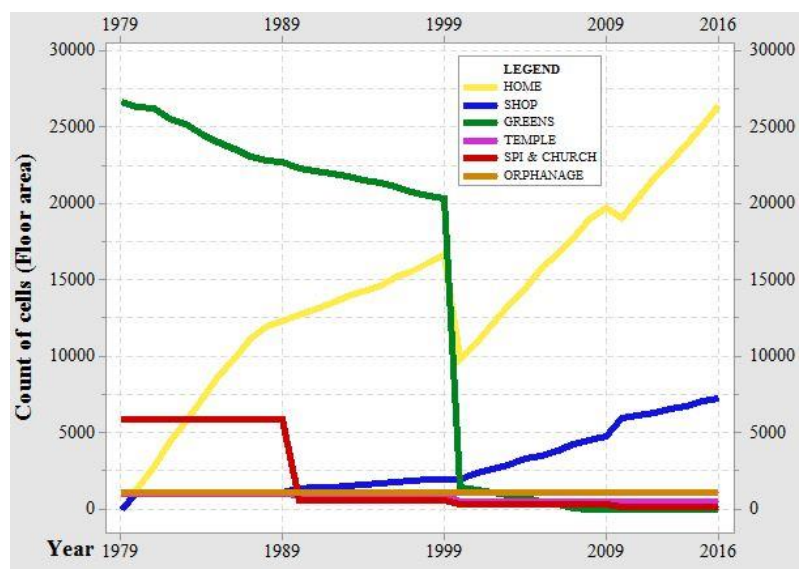

Figure 8: Graph of scenario 2's time-series plot for each spatial unit's floor areas (Year 1979-2016). Source: Authors. 
similar rate for both scenarios. Nonetheless, the number of orange (orphanage) remained static for scenario 2 (Figure 8). Based on this qualitative observation and quantitative analysis, we can suggest that this hypothesis was probably not the main driving force responsible for the expansion of homes across the informal settlement.

\subsubsection{STATISTICAL COMPARISON}

We now turn our attention to a statistical comparison of the simulation results from scenario 1 and 2 at the end of the simulation trials. Each simulation trial was repeated 20 times. The results are listed in Table 1. We have tested the following null hypotheses: there was no significant difference between the median values of the homes/shops/orphanage in scenario1 and scenario 2. The result of a Wilcoxon Rank sum test $(p<0.01)$ confirms that there are no significant differences in the number of homes and/or shops at the end of the simulations between scenarios. However, there was a statistically significant difference for the orphanage. It should be noted that the evolutionary trajectory of each scenario is significantly different (see time series plots in Figure 7 and 8). The qualitative patterns observed in the output from scenario 1 is consistent with observations of the case study site.

Table 1: Statistic data from the spatial units' count of the cells: homes, shops and orphanage generated from model.

\begin{tabular}{cccccc}
$\begin{array}{c}\text { SPATIAL } \\
\text { UNIT }\end{array}$ & SCENARIO & $\begin{array}{c}\text { SAMPLE } \\
\text { SIZE }\end{array}$ & MEDIAN & MEAN & STDEV \\
\hline \multirow{2}{*}{ HOMES } & 1 & 20 & $\mathbf{2 3 3 0 9}$ & 23666 & 608 \\
& 2 & 20 & $\mathbf{2 5 4 7 9}$ & 25835 & 740 \\
\hline SHOPS & 1 & 20 & $\mathbf{6 4 3 6}$ & 6476 & 300 \\
& 2 & 20 & $\mathbf{7 2 4 4}$ & 7470 & 509 \\
\hline & 1 & 20 & $\mathbf{3 1 7 8}$ & 3171 & 98 \\
ORPHANAGE & 2 & 20 & $\mathbf{1 1 0 4}$ & 1104 & $2.33 \mathrm{E}-13$
\end{tabular}

\subsection{FINDINGS AND DISCUSSIONS}

In this paper, we have introduced an $A B M$ - a virtual environment - to explore the urban morphology of TmorDa. An inspection of the simulation running, clearly shows transitions between built forms: the state of the cubes changed from red (church) and magenta (Chinese temple) to yellow (homes) then to blue (shops), at the same location. Yellow or blue cubes increased in number, expanding to occupy the entire area, often over the residual spatial units.

Our simulation results provide supporting evidence that the hypotheses presented as possible driving forces of morphological change derived from field work are plausible. The model succeeded in simulating the formation of the informal settlement, given the constraint of limited empirical datasets. For example, population levels and growth rate over time for Tmor-Da were not known. Therefore, we created a synthetic population of 1000 people with a per annum growth rate of $3 \%$. As a result, the simulation could be used to model the growth of the population as shown in Figure 4 (Top-left: Time plot of total population vs home capacity graph).

It is important to note that our agent-based simulation model is very sensitive to model parameters and transition rules. Small variations to transitional rules and parameters produced very different trajectories - a behaviour to be expected in any CAS model. This type of ABM can play an essential role in helping to explain the CAS exhibited in 
informal settlements. ABM can assist in understanding more about adaptable and resilient urban structures.

The possibility of running the simulations for a larger number of time steps presents an opportunity to trace possible paths that the morphological changes may take. However, there is a need to expand the current model by incorporating coupled social-urban datasets, which would allow for fine-grained micro-scaled urban dynamics to be explored. One interesting direction would be to integrate pedestrian movements and examine the effects of such movements on the emergence of urban nodes.

In conclusion, the ABM model of evolutionary dynamics of Tmor-Da designed and evaluated in this study has contributed to the establishment of a systematic method to analyse the urban evolution of informal settlements. Finally, the model served as an investigative tool to examine the most plausible causes of an informal settlement's development, derived from fieldwork observations.

\section{REFERENCES}

Barros J., Sobreira F. (2008) City of Slums: self-organisation across scales. In: Minai A.A., Bar-Yam Y. (eds) Unifying Themes in Complex Systems IV. Springer, Berlin, Heidelberg

Batty, M., \& Longley, P. (1994). Fractal cities : a geometry of form and function. Academic Press. Retrieved from http://webcache.googleusercontent.com/search?q=cache:2Oi YyDm11BYJ:www.fractalcities.org/book/Fractal\%2520Cities $\% 2520$ Introduction. $p d f+\& c d=4 \& h l=e n \& c t=c l n k \& g l=a u$

Dibble, J., Prelorendjos, A., Romice, O., Zanella, M., Strano, E., Pagel, M., \& Porta, S. (2015). Urban Morphometrics: Towards a Science of Urban Evolution. Retrieved from https://arxiv.org/abs/1506.04875

Fisher-Gewirtzman, D., \& Blumenfeld-Liberthal, E. (2012). An agent-based model for simulating urban morphology: Sachnin as a case study. Survey Review, 44(325), 162-167. https://doi.org/10.1179/1752270612Y.0000000001

Holland, J. H. (2010). Complex Adaptive Systems. Daedalus, 121(1), 17-30. https://doi.org/10.1007/3-540-26869-3_16

Karakiewicz, J. (2016). Interventions in Complex Urban Systems: How to Enable Modeling to Account for Disruptive Innovation (pp. 113-127). https://doi.org/10.1007/978-3-319-30178-5_8

Marcos, I. (2012). Urban morphogenesis. Semiotica, 2012(192), 1-14. https://doi.org/10.1515/sem-2012-0077

Habraken, N. J. (1998). The structure of the ordinary: form and control in the built environment. Cambridge, Mass.: MIT Press.

Oliveira, V. (2016). Urban Morphology. Cham: Springer International Publishing. https://doi.org/10.1007/978-3-31932083-0

Partanen, J. (2017). An urban cellular automata model for simulating dynamic states on a local scale. Entropy, 19(1). https://doi.org/10.3390/e19010012

Patel, A., Crooks, A., \& Koizumi, N. (2018). Spatial Agent-based Modeling to Explore Slum Formation Dynamics in Ahmedabad, India (pp. 121-141). Springer, Cham. https://doi.org/10.1007/978-3-319-59511-5_8

Patel, S., \& Baptist, C. (2012). Editorial: Documenting by the undocumented. Environment and Urbanization, 24(1), 3-12. https://doi.org/10.1177/0956247812438364

Sharpe, C. A. (1986). THE TEACHING OF URBAN MORPHOGENESIS. The Canadian Geographer/Le G?ographe Canadien, 30(1), 53-59. https://doi.org/10.1111/j.1541-0064.1986.tb01025.x

Vanegas, C. a., Garcia-Dorado, I., Aliaga, D. G., Benes, B., \& Waddell, P. (2012). Inverse design of urban procedural models. ACM Transactions on Graphics, 31(6), 1. https://doi.org/10.1145/2366145.2366187

Venerandi, A., Zanella, M., Romice, O., Dibble, J., \& Porta, S. (2016). Form and urban change - An urban morphometric study of five gentrified neighbourhoods in London. Environment and Planning B: Planning and Design. https://doi.org/10.1177/026581351665803 